\title{
The Role of Systematic Data Reviews in Safety Assessment of Infant Nutrition
}

\author{
Hania Szajewska \\ Department of Paediatrics, The Medical University of Warsaw, Warsaw, Poland
}

\author{
Key Words \\ Systematic review $\cdot$ Safety $\cdot$ Tolerance $\cdot$ Adverse effects • \\ Pediatrics
}

\begin{abstract}
To make informed, evidence-based decisions regarding whether a given intervention is necessary, information about its benefits as well as harms is needed. Systematic reviews are always at the top of the evidence hierarchy. In general, the methodology for conducting systematic reviews of benefits and safety/harms overlaps. While systematic reviews of randomized controlled trials (RCTs) are sufficient for providing information regarding the efficacy and short-term safety aspects of these trials, they may be insufficient for providing adequate information about long-term safety and consequences. To adequately address harms, systematic reviews should include evidence from both RCTs and non-RCTs. The inclusion of the latter is to be considered particularly for addressing rare adverse effects, long-term adverse effects, or outcomes that were unknown at the time the RCTs were performed.

Copyright $\odot 2012$ S. Karger AG, Basel
\end{abstract}

\section{Introduction}

Should probiotics be given routinely to preterm infants to prevent necrotizing enterocolitis? Does docosahexaenoic acid intake contribute to the visual develop- ment of infants up to 12 months of age? Should protein hydrolysates be given for allergy prevention to all infants regardless of the risk of allergy? Are those interventions likely to be more beneficial than harmful? To make informed, evidence-based decisions regarding whether a given intervention is necessary, information about its benefits as well as harms is needed.

Regardless of the question being asked, evidencebased medicine locates systematic reviews at the top of the evidence hierarchy. The methodology for conducting systematic reviews of interventions has been developed by the Cochrane Collaboration and is well established [1]. Although the basic principles are the same regardless of the benefits or harms that are being evaluated and regardless of the type of intervention being studied (e.g. pharmacological, nutritional, etc.), a number of methodological issues need to be taken into account when harms are addressed. This article discusses issues related to the conduct of systematic reviews in assessing harms.

\section{Terminology}

\section{Safety or Harms}

Terms such as 'adverse effect', 'adverse event', 'side effect', 'safety', and 'toxicity' are commonly used interchangeably when reporting harms-related issues, even when there is a distinction between those terms (see table 1 for selected definitions). Members of the CONSORT Group encourage the use of the term 'harms' instead of

\section{KARGER \\ Fax +4161306 1234 \\ E-Mail karger@karger.ch}

www.karger.com
(C) 2012 S. Karger AG, Basel

0250-6807/12/0603-0204\$38.00/0

Accessible online at:

www.karger.com/anm
Hania Szajewska, MD

Department of Paediatrics, The Medical University of Warsaw Dzialdowska 1

PL-01-184 Warsaw (Poland)

Tel. +4822452 33 09, E-Mail hania@ipgate.pl 
Table 1. Terminology and definitions (based on [2, 11])

\begin{tabular}{|c|c|}
\hline Term & Definition \\
\hline Adverse effect & $\begin{array}{l}\text { An adverse event for which the causal relation } \\
\text { between the drug/intervention and the event } \\
\text { is at least a reasonable possibility. The term } \\
\text { 'adverse effect' applies to all interventions, while } \\
\text { 'adverse drug reaction' is used only with drugs. } \\
\text { In the case of drugs, an adverse effect tends to } \\
\text { be seen from the point of view of the drug and } \\
\text { an adverse reaction is seen from the point of } \\
\text { view of the patient. }\end{array}$ \\
\hline Adverse event & $\begin{array}{l}\text { An adverse outcome that occurs during or after } \\
\text { the use of a drug or other intervention but is not } \\
\text { necessarily caused by it. }\end{array}$ \\
\hline $\begin{array}{l}\text { Adverse drug } \\
\text { reaction }\end{array}$ & An adverse effect specific to a drug. \\
\hline Harms & $\begin{array}{l}\text { The totality of possible adverse consequences of } \\
\text { an intervention or therapy; they are the direct } \\
\text { opposite of benefits, against which they must be } \\
\text { compared. }\end{array}$ \\
\hline Safety & $\begin{array}{l}\text { Substantive evidence of an absence of harm. } \\
\text { The term is often misused when there is } \\
\text { simply absence of evidence of harm. }\end{array}$ \\
\hline Side effect & $\begin{array}{l}\text { Any unintended effect, adverse or beneficial, of } \\
\text { an intervention. Side effects are most } \\
\text { commonly associated with pharmaceutical } \\
\text { products, in which case they are related to the } \\
\text { pharmacological properties of the drug at doses } \\
\text { normally used for therapeutic purposes in } \\
\text { humans. }\end{array}$ \\
\hline Toxic effect & Describes drug-related harms. \\
\hline Complication & $\begin{array}{l}\text { Describes surgical or other invasive harms of } \\
\text { interventions. }\end{array}$ \\
\hline
\end{tabular}

'safety' [2]. The rationale for this is that the term 'safety' provides reassurance obscuring the real or potential 'harms' that an intervention may cause.

\section{Systematic Review and Meta-Analysis}

According to the Cochrane Collaboration, a systematic review is 'a review of a clearly formulated question that uses systematic and explicit methods to identify, select and critically appraise relevant research, and to collect and analyze data from studies that are included in the review. Statistical methods may or may not be used to analyze and summarize the results of the included trials' [1]. A meta-analysis is a name that is given to any review article when statistical techniques are used in a systematic review to combine the results of included trials to produce a single estimate of the effect of a particular intervention (i.e. a number or a graph) [1].

\section{How Does One Conduct a Systematic Review?}

In brief, the following five steps are needed to conduct a systematic review: (1) formulation of the review question (the problem); (2) searching, i.e. locating and selecting controlled trials based on predefined inclusion and exclusion criteria; (3) selecting studies and collecting data; (4) assessment of the risk of bias in the included trials, and (5) analyzing the data and presenting the results. Some guidance for peculiarities of conducting systematic reviews of adverse effects is available from the Cochrane Collaboration [3]. Even if limited, there is literature on systematic reviews of adverse effects of pharmaceutical interventions; methodological issues related to nutritional trials are rarely discussed separately. Depending on the research question, systematic reviews may include randomized controlled trials (RCTs) and non-RCTs. Only the methodology for the development of RCTs is well developed.

\section{Reporting of Harms}

A survey of the methods used in systematic reviews in which the primary outcome was an adverse effect or the effects of health care interventions (primarily pharmacological) revealed a number of methodological limitations; these included insufficient information to reproduce the search strategies, no assessment of the quality of the included trials, no consideration of the heterogeneity of the trials, and a lack of information about the source of the findings. It was concluded that improvement in the methodology and reporting of systematic reviews of adverse effects is needed [4].

\section{Issues Related to the Conduct of Systematic Reviews}

\section{Research Question}

As stated earlier, the initial step in the review process is the formulation of a research question. The key components of a research question should address the types of participants, interventions, comparisons, and outcomes of interest. 
A research question about safety/harms may have a broad or narrow focus. A broad focus, such as 'to review the published evidence related to the safety and health effects of the administration of formulae supplemented with probiotics and/or prebiotics compared with unsupplemented formulae' [5], provides a broad overall general picture of potential problems, both known and previously unrecognized. Still, the latter may be better detected through primary surveillance [6]. Data collection and extraction may be difficult as well as time and resource intensive. In contrast, a narrow focus of the research question (e.g. 'Does Lactobacillus GG increase the risk of sepsis in infants?') allows evaluation of only a selected, known-in-advance, but usually potentially serious adverse effect. This is the easiest approach, but it does not provide a complete safety or tolerability overview. The conclusions are limited.

\section{Types of Studies}

The main objective and research question will guide the decision regarding what types of studies to be included in the systematic review. For pharmacological interventions, Loke et al. [7] recently proposed a structured framework for study selection when undertaking comprehensive evaluation of adverse effects, which in general can be applied to other interventions. In brief, systematic reviews should be based on two pillars of evidence. For potential benefits, the most reliable evidence comes from RCTs; for adverse effects, a considerable synthesis of evidence from RCTs and non-randomized trials is required. Safety questions - specifically those related to long-term or rare adverse effects - are unlikely to be answered by RCTs. The inclusion of non-randomized studies, such as cohort studies, case-control studies, case reports or case series, is to be considered for addressing rare adverse effects, long-term adverse effects, or outcomes that were unknown at the time the RCTs were performed. Additional support for including a broad range of studies when evaluating adverse effects comes from a recent report by Golder et al. [8]. The findings from this study documented that there is no significant difference on average between estimates of the risk of adverse effects from meta-analyses of RCTs and of observational studies. Thus, it suggests that limiting systematic reviews of adverse effects to certain study designs is inappropriate and a more broad approach is needed. One of the major problems concerning the evaluation of harm, particularly when evaluating rare adverse effects, is a type II error, i.e. a conclusion that there is no evidence of harm, when it actually does cause unintended adverse effects [7].

\section{Search Strategy}

Efficient search strategies for identifying reports of adverse effects in MEDLINE and EMBASE have yet to be developed. Until this happens, it is recommended that one uses a combination of index terms (such as Medical Subject Headings (MeSH) in MEDLINE and EMTREE in EMBASE) and subheadings. For example, 'probiotics' is the MeSH term and 'adverse effects' is the subheading. However, no single index or subheading search term can reliably identify all data on adverse effects.

\section{Additional Data Sources}

In addition to bibliographic databases (e.g. MEDLINE, EMBASE, CENTRAL), information regarding food safety, tolerability, and adverse effects can be identified from product information sheets, websites of regulatory authorities (e.g. European Food Safety Authority, Food and Drug Administration), or companies manufacturing nutritional products for infants and children. One major problem with these sources of information is the lack of simple or systematic methods used for identifying information on harm.

\section{Unpublished Data}

The inclusion of unpublished data is controversial. One important argument is that unpublished studies differ systematically from those that have been published. Particular concern is with regard to inadequate reporting of adverse events. The nonpublication of a trial (and thus noninclusion in the systematic review) can lead to false assumptions regarding the efficacy of the treatment [9].

\section{Assessing Risk of Bias}

In clinical trials, usually the following criteria generally associated with good-quality studies recommended by the Cochrane Collaboration are evaluated: adequacy of sequence generation, allocation concealment, and blinding of investigators, participants, outcome assessors, and data analysts; intention-to-treat analysis; and comprehensive follow-up ( $\geq 80 \%$ ). For adverse effects, the areas of concern include the following: (1) methods for monitoring and detecting adverse effects (e.g. active compared with passive surveillance leads to more recorded adverse effects); (2) conflicting interests (e.g. some sponsors/investigators may withhold unfavorable trial data or may not make full trial reports available); (3) selective outcome reporting (on average, more than onethird of efficacy outcomes and one half of harm outcomes in parallel-group trials were inadequately reported; the proportions were much higher in crossover studies due to 
unreported paired data); and (4) blinding. For non-RCTs, there is an absence of a defined tool for assessing risk of bias. One report revealed 115 different quality assessment tools used for non-randomized intervention studies [10].

\section{Conclusions}

Systematic reviews are always at the top of the evidence hierarchy. In general, the methodology for conducting systematic reviews of benefits and safety/harms overlaps. While systematic reviews of RCTs are sufficient for providing information regarding the efficacy and short-term safety aspects of these trials, they may be in- sufficient for providing adequate information about longterm safety and consequences. To adequately address harms, systematic reviews should include evidence from both RCTs and non-RCTs. The inclusion of the latter is to be considered particularly for addressing rare adverse effects, long-term adverse effects, or outcomes that were unknown at the time the RCTs were performed.

\section{Disclosure Statement}

No conflict of interest to declare.

\section{References}

1 Higgins JP, Green S (eds): Cochrane Handbook for Systematic Reviews of Interventions Version 5.0.1 (updated September 2008) The Cochrane Collaboration, 2008. www. cochrane-handbook.org.

2 Ioannidis JP, Evans SJ, Gøtzsche PC, O’Neill RT, Altman DG, Schulz K, Moher D; CONSORT Group: Better reporting of harms in randomized trials: an extension of the CONSORT statement. Ann Intern Med 2004;141:781-788.

3 Loke YK, Price D, Herxheimer A; on behalf of the Cochrane Adverse Effects Methods Group: in Higgins JP, Green S (eds): Cochrane Handbook for Systematic Reviews of Interventions. Wiley-Blackwell, 2009, pp 433-447.
4 Golder S, Loke Y, McIntosh HM: Room for improvement? A survey of the methods used in systematic reviews of adverse effects. BMC Med Res Methodol 2006;6:3.

-5 Braegger C, Chmielewska A, Decsi T, Kolacek S, Mihatsch W, Moreno L, Pieścik M, Puntis J, Shamir R, Szajewska H, Turck D, van Goudoever J; ESPGHAN Committee on Nutrition: Supplementation of infant formula with probiotics and/or prebiotics: a systematic review and comment by the ESPGHAN committee on nutrition. J Pediatr Gastroenterol Nutr 2011;52:238-250.

6 Loke YK: Assessing the benefit-harm balance at the bedside. BMJ 2004;329:7-8.

7 Loke YK, Golder SP, Vandenbroucke JP: Comprehensive evaluations of the adverse effects of drugs: importance of appropriate study selection and data sources. Ther Adv Drug Saf 2011;2:59-68.
8 Golder S, Loke YK, Bland M: Meta-analyses of adverse effects data derived from randomised controlled trials as compared to observational studies: methodological overview. PLoS Med 2011;8:e1001026.

$\checkmark 9$ Whittington CJ, Kendall T, Fonagy P, Cottrell D, Cotgrove A, Boddington E: Selective serotonin reuptake inhibitors in childhood depression: systematic review of published versus unpublished data. Lancet 2004;363: 1341-1345.

10 Dinnes J, Sowden AJ, Deeks J, Altman D: A review of quality assessment tools for nonrandomised intervention studies. Annual Meeting of the International Society of Technology Assessment in Health Care. Philadelphia, 2001.

11 https://www.cebp.nl/media/m69.pdf (accessed on 3 May 2011). 